\section{ADWE-10 RISK FACTORS FOR LINE INFECTIONS IN A} CAMBRIDGE HPN COHORT

C Adgey, T Raine, H Lawrence, LM Sharkey, JM Woodward, DCO Massey. Addenbrookes Hospital, Cambridge, UK

\subsection{6/gutjnl-2018-BSGAbstracts. 12}

Introduction Risk factors for central line-associated blood stream infections (CLABSI) were investigated in a home parenteral nutrition (HPN) cohort.

Methods All patients receiving HPN between January 1st 2016 and July 31st 2017 were included. Data was analysed by individual central venous catheters (CVC) rather than by patients who may have had multiple CVCs. CLABSI (fever, positive blood cultures through line, no alternative infection source) was considered an end point even if the line was salvaged. Patient factors included underlying diagnosis, presence of end stoma, enterostomy, use of opioids/Tauralock and line type. Infection-free line survival was estimated by the KaplanMeier method. Univariate and multivariate Cox proportionalhazards models were used to determine the contribution of baseline variables.

Results 251 CVCs were followed in 142 patients $(67.6 \%$ female, age 17-92, median 56.5) over 94779 line days. Over the 18 month period 49 CLABSI were documented in 37 patients, equating to 0.52 infections per 1000 catheter days.

Cox proportional-hazards modelling revealed a number of individual factors associated with decreased CLABSI-free line survival. Patients with joint hypermobility and GI symptoms (OR 14.6, 95\% CI 3.16-68.1, p<0.001); enteric dysmotility (OR 7.1, 95\% CI 1.5-32.4, $\mathrm{p}=0.01$ ) and use of a dual lumen line (OR 2.26, 95\% CI 1.24-4.1, $\mathrm{p}=0.007)$ were also associated with increased risk of CLABSI. Other variables showing an association with increased risk of CLABSI included use of opioids $(\mathrm{p}<0.01)$, use of tauralock $(\mathrm{p}<0.001)$ and presence of a feeding or venting gastrostomy tube $(\mathrm{p}<0.01)$. Presence of an end stoma or a fistula was associated with decreased risk of CLABSI $(\mathrm{p}<0.01)$. Increasing age was associated with decreased risk of CLABSI (OR 0.96 for each additional year of life, 95\% CI 0.94 $0.97, \mathrm{p}<0.001)$.

We built a multivariate hazards model incorporating variables showing a significant correlation with CLABSI in univariate analysis. Age and diagnosis remained significant; several of the variables showed strong co-linearity. An underlying diagnosis of joint hypermobility and GI symptoms was significantly associated with age in the lowest quartile. Since age and diagnosis were confounded, we tested the model with each in isolation and found that a model using diagnosis fitted the data better than a model using age (model concordance 0.815 for diagnosis vs 0.804 for age, $p<0.0001$ for both).

Conclusion We examined the effect of patient factors on CLABSI in our HPN cohort using a novel line-focussed approach. Our overall infection rates are comparable with contemporary case series. Opioid use was confirmed as a risk factor, but presence of an end stoma was not. Notably young patients with joint hypermobility and GI symptoms comprised a high risk group for line infection.

\section{ADTH-01 ENDOSCOPIC FULL THICKNESS RESECTION IN THE COLON: 3-YEAR MULTICENTRE UK EXPERIENCE}

${ }^{1}$ Max Hu*, ${ }^{1}$ Imdadur Rahman, ${ }^{2}$ Sauid Ishaq, ${ }^{3}$ Pradeep Bhandari, ${ }^{4}$ Stirling Pugh, ${ }^{5}$ Susi Green, ${ }^{5,6}$ Noriko Suzuki, ${ }^{7}$ Andrew Hopper, ${ }^{8}$ John Martin, ${ }^{6}$ Siwan Thomas-Gibson, ${ }^{1}$ Praful Patel, 'Phil Boger. 'University Hospital Southampton, Southampton, UK; ${ }^{2}$ Russells Hall Hospital, Dudley, UK; ${ }^{3}$ Queen Alexandra Hospital, Portsmouth, UK; ${ }^{4}$ Musgrove Park Hospital, Taunton, UK; ${ }^{5}$ Brighton \& Sussex University Hospital, Brighton, UK; ${ }^{6}$ St Marks Hospital, London, UK; ${ }^{7}$ Royal Hallamshire Hospital, Sheffield, UK; ${ }^{8}$ Charing Cross Hospital, London, UK

\subsection{6/gutjnl-2018-BSGAbstracts. 13}

Introduction Endoscopic full thickness resection (eFTR) of the colon using the full thickness resection device (FTRD) is a novel method for removing lesions involving, or tethered to, deeper layers of the colonic wall. The UK FTRD registry collects data from multiple centres performing this procedure. We describe the feasibility and early outcomes of this technique.

Methods Registry data from April 2015 - January 2018 was analysed. Main outcome measures were technical success, procedural time, specimen size, R0 resection, endoscopic clearance, and adverse events. Reported technical difficulties were collated.

Results 38 cases were performed across 8 centres (median 2 cases per centre, range 1-23). Mean patient age was 70 years (39-93). Indications for eFTR include non-lifting adenoma (18 cases), T1 tumour resection (10), submucosal tumour (7), and appendix base adenoma (3).

In $97.4 \%(37 / 38)$ of patients the lesion was reached with the FTRD. One caecal lesion could not be reached due to sigmoid diverticulosis. The procedure was technically successful in $91.9 \%$ of patients (34/37). Median procedure time was 41 minutes (11-86), median resection time 6 minutes (2-36), and median specimen size $22 \mathrm{~mm}(10-30)$. R0 resection was achieved in $76.5 \%$ of patients (26/34). R0 resection was not achieved in 8 patients, of which 5 had no residual lesion on follow up, giving a total endoscopic clearance rate of $91.2 \%$ (31/34).

Technical difficulty occurred in 9 patients; 6 due to snare failure and 3 due to lesion slippage on clip deployment. Of these 9 cases, 7 achieved R0 resection by use of further snare. Of the 2 patients with R1 resection, one has had follow up at 4 months with no evidence of residual lesion.

Resection was unsuccessful in 3 patients; 2 due to significant tethering restricting lesion capture, and one due to haemodynamic instability from atrial fibrillation whilst pulling lesion into cap.

Complications occurred in 2 patients; one acute appendicitis at day 6 after resection of appendix base adenoma, and one with mild asymptomatic stricture at eFTR site at follow up. There were no cases of bleeding, perforation, or fistula.

Conclusions eFTR has a high success rate in treating lesions not previously amenable to endoscopic therapy. Whilst technical difficulties may arise; complication rates are low and outcomes acceptable, making eFTR a viable alternative to surgery. 